\begin{tabular}{|c|l|}
\hline Title & $\begin{array}{l}\text { A parameter study of pencil beam proton dose distributions for the treatment of ocular mel anoma utilizing spot } \\
\text { scanning }\end{array}$ \\
\hline Author(s) & $\begin{array}{l}\text { Sutherland, Kenneth; Miyajima, Satoshi; Date, Hiroy uki; Shirato, Hiroki; Ishikawa, Masay ori; Murakami, Masao; } \\
\text { Yamagiwa, Mitsuru; Bolton, Paul; Tajima, Toshiki }\end{array}$ \\
\hline Citation & $\begin{array}{l}\text { Radiological Physics and Technology, 3(1), 16-22 } \\
\text { https:/doi.org/10.1007/312194_009-0071-4 }\end{array}$ \\
\hline Issue Date & 2010-01 \\
\hline Doc URL & http://hdl.handle.net/2115/46897 \\
\hline Rights & The original final publication is avail lable at www.springerlink.com \\
\hline Type & article (author version) \\
\hline File Information & RPT31_16-22.pdf \\
\hline
\end{tabular}

Instructions for use 
Kenneth Sutherland, Satoshi Miyajima, Hiroyuki Date, Hiroki Shirato, Masayori Ishikawa, Masao Murakami, Mitsuru Yamagiwa, Paul Bolton, and Toshiki Tajima

\title{
A parameter study of pencil beam proton dose distributions for the treatment of ocular melanoma utilizing spot scanning
}

\author{
K. Sutherland, H. Date, H. Shirato, M. Ishikawa \\ Hokkaido University School of Medicine, Sapporo-shi Kita-ku Kita 12 Jo Nishi 5 \\ Chome 060-0812, Japan \\ K. Sutherland, S. Miyajima \\ Japan Science and Technology, Saitama-ken Kawaguchi-shi Motomachi 4 Chome 1 Ban \\ 8 Go 332-0012, Japan \\ M. Yamagiwa, P. Bolton, T. Tajima \\ Photo-Medical Research Center, Japan Atomic Energy Agency, Kizugawa, Kyoto 619- \\ 0215, Japan \\ M. Murakami \\ Hyogo Ion Beam Medical Center, Shinguu Chou Hikarimiya 1-2-1Tatsuno City, Hyogo \\ Prefecture, Japan
}

Corresponding author:

Kenneth Sutherland

e-mail: kensuth@med.hokudai.ac.jp

phone: +81-11-706-7638

fax: $+81-11-706-7639$ 
Abstract Results of Monte Carlo calculated dose distributions of proton treatment of ocular melanoma are presented. An efficient spot-scanning method utilizing active energy modulation which also minimizes the number of target spots was developed. We simulated various parameter values for the particle energy spread and the pencil-beam diameter in order to determine values suitable for medical treatment. We found that a 2.5 -mm-diameter proton beam with a 5\% Gaussian energy spread is suitable for treatment of ocular melanoma while preserving vision for the typical case that we simulated. The energy spectra and required proton current were also calculated and are reported. The results are intended to serve as a guideline for a new class of low-cost, compact accelerators.

Key words; proton therapy, ocular melanoma, Monte Carlo simulation, laser acceleration 


\section{Introduction}

Proton beams have the potential to decrease normal tissue damage and allow dose escalation in cancer therapy because the beam profile allows a more localized dose distribution at the tumor than do traditional X-rays. For covering the volume of a target lesion in particle therapy for cancer, two methods have been employed: passive scattering and spot scanning. In the passive scattering method, secondary neutrons from scatter foils, compensators, and collimators are a possible source of secondary malignancy [1]. Spot scanning was first proposed as an alternative to passive scattering methods by Kanai et al. [2] and was further investigated by Lomax et al. [3]. Spot scanning utilizes magnetic and mechanical scanning of a pencil proton beam such that individually weighted Bragg peaks are distributed under computer control [4]. For spot scanning, there is no need for patient-specific collimators, thereby reducing the whole-body neutron dose to the patient. Another advantage is that most of the particles from the accelerator can be delivered to the patient, rather than being absorbed by collimators or compensators, and therefore this method is potentially more efficient.

In this work, we present results of Monte Carlo simulations of proton dose distributions in which we used parameterized proton beams applied to ocular melanoma. We hope that the results of this study will serve as a guide for researchers developing proton facilities for medical treatment. We comment on the potential relevance of laser-accelerated protons [5-8] for the treatment of ocular melanoma, which requires lower proton energies than do more deeply seated tumors, as well as relatively lower doses (fewer protons) because such tumors are typically small. However, the results should be applicable to proton therapy in general. 


\section{Materials and Methods}

\subsection{Monte Carlo simulation speed improvements}

Geant4 [9] version 8.0p1 was used for these simulations. Geant4 has been validated previously for medical-physics applications [10]. In order to improve the execution speed, we modified the particle navigation library following Jiang and Paganetti [11]. To improve efficiency on a PC cluster, we also developed a custom parallelization of Geant4 [12]. Simulations yield exactly the same results when running in parallel on a cluster or on a single processor as long as random-number-generator seed values are maintained and are set at the beginning of each event.

Following Jiang and Paganetti [11], four physics processes were registered to the Geant4 physics list for proton interactions: proton elastic scattering (G4HadronElasticProcess), proton inelastic scattering (G4HadronInelasticProcess), ionization (G4hLowEnergyIonisation), and multiple scattering (G4MultipleScattering). For improved efficiency, only secondary protons and neutrons were tracked. The energy from secondary electrons was deposited locally because the range was assumed to be less than $1 \mathrm{~mm}$ in water. The Geant4 maximum step size was limited to $1 \mathrm{~mm}$.

\subsection{Radiation treatment simulation software}

We developed an application which investigates the effects of the proton beam diameter and energy spread on the dose distribution. The software allows the user to open a series of DICOM CT images, specify the target volume, one or more gantry positions, and specify various beam characteristics. The user can also enter the particle (i.e., event) count. We chose a particle count of 1 million for this study, which we determined to be sufficient for good energy deposit distribution statistics with a reasonable processing time. 
We used a series of $11 \mathrm{CT}$ images of a disease-free human head with a slice thickness of 2.5 $\mathrm{mm}$ and a pixel spacing of $0.3125 \mathrm{~mm}$. The CT pixel value (in Hounsfield units) of each voxel was used for determining the voxel material. Each material is assigned a density and a chemical composition according to the data provided by Schneider et al. [13]. The software generates files which specify the different voxel materials and an event list containing the initial source position, direction, and energy of each particle in the simulation. The dose accumulation grid had the same size and dimensions as the CT data: $512 \times 512 \times 11$; i.e. we did not subsample or smooth the CT values.

A database of depth and lateral dose profile curves was pre-computed by use of the Monte Carlo package Particle and Heavy Ion Transport Code System (PHITS) [14, 15]. This database was used by our planning software for determining the initial energy peak, energy spread, and spot spacing. Depth-dose curves were computed for proton beams incident on water with energies from $30 \mathrm{MeV}$ to $250 \mathrm{MeV}$ in $1-\mathrm{MeV}$ increments. Four values of the Gaussian energy spread were computed: $0 \%, 5 \%, 10 \%$, and $15 \%$, at a depth resolution of 0.1 $\mathrm{mm}$. Lateral offset tables were computed for energies from $30 \mathrm{MeV}$ to $200 \mathrm{MeV}$ in $1-\mathrm{MeV}$ increments, with the use of the same energy spread values, $0 \%, 5 \%, 10 \%$, and $15 \%$, and with beam diameters of $0,1.25,2.5,5$, and $10 \mathrm{~mm}$. The tables were stored in a binary format which minimized the time necessary for reading of the data by the planning software.

An initial weighting factor was assigned to each target spot, which was used for determining the particle count. The deepest spot (associated with the highest energy) along a beam is assigned weight 1.0. Shallower spots are then assigned weights less than 1.0 based on the precomputed database of dose distribution curves in order to achieve a spread-out Bragg peak (SOBP). The target weight is then used with the total particle count for assignment of individual particle counts for each target spot. At this step, the energy spread and beam 
diameter are factored in by addition of small random values to the initial energy and position of each particle. In the case of the energy spread, random numbers are chosen so that the resulting particle energies have a Gaussian distribution with the specified full width at half maximum (FWHM). Another pair of random numbers is chosen to place the particle within the specified beam diameter. Particles are distributed evenly along the beam axis. The particle list is then written to a file which is read by the simulation program.

\subsection{Target spot spacing}

We incorporated a spot scanning method where the beam position and direction are fixed while target spots along the beam direction are scanned by means of depth variation; i.e., active energy variation for depth modulation. All beams are assumed to be parallel to each other in this simulation (Fig. 1). This method requires a rapid alteration of the proton energy.

Our software also has the ability automatically to place target spots at locations with variable spacing based on the pre-computed database of dose profile curves in water. In the case of lateral spacing, lateral fall-off curves at the beam's pre-computed Bragg peak depth are used for determining the width (FWHM) of the beam, specifying a "spot width". The spot width is mostly affected by the beam diameter and by lateral scatter. For depth spacing, the beam's pre-computed depth-dose profile curve was used similarly for specifying a "spot depth". The spot depth is affected mostly by the energy spread.

Utilizing spot width and depth alone for spot spacing results in an uneven dose distribution within the target region due to under-dosed regions between spots. For achieving a smooth dose distribution, the spot width and the spot depth are multiplied by a "spacing factor". The spacing factor is usually less than 1.0 and has the effect of placing the spots closer together, i.e., increasing the number of spots. A spacing factor of 0.5 , which we found yields a 
relatively smooth dose distribution while minimizing the number of target spots, was used throughout this simulation. A more detailed examination of the effect of the spacing factor on the dose distribution and the number of spots is a topic for future investigation.

\subsection{Dose distribution optimization}

It is difficult to predict the exact dose distribution in inhomogeneous patient volumes based on CT data alone. Therefore, after the initial Monte Carlo simulation, we fine-tuned the particle counts assigned to each target spot in the following way. The dose distribution for each target spot is calculated with the Monte Carlo simulation program and stored in separate files, one dose distribution file per target spot. The individual files are read and summed to form a complete dose distribution. The dose deposited at each target spot is compared with the dose average in the planning target volume (PTV). Spots that received less than the average dose (cold spots) are assigned more particles, and spots with a higher dose (hot spots) are assigned fewer particles. The process is repeated iteratively until it is determined that the result cannot be optimized further. Note that we do not attempt to reduce the dose deposited in critical structures; we only attempt to achieve a uniform dose distribution within the target volume in this optimization process.

\subsection{Target polygon}

The gross target volume (GTV) was modeled as a semi-ellipsoid with a semi-sphere base of height $4.8 \mathrm{~mm}$ and basal diameter $13 \mathrm{~mm}$. The minimum tumor-optic disk distance was $5 \mathrm{~mm}$. The tumor-macula distance was $4 \mathrm{~mm}$. The values were chosen to represent a typical tumor based on data reported by Dendale et al. [16]. The GTV was generated in the planning software by specification of the tumor height, base diameter, eye center, and tumor base position. A $2 \mathrm{~mm}$ margin was automatically added to the perimeter of the GTV to form the 
PTV. The volume of the PTV was $.734 \mathrm{~cm}^{3}$. The target polygon (PTV) and organs at risk (OARs) are shown in Fig. 2.

The prescribed dose was set to $54.5 \mathrm{~Gy}$, which is equal to 60 cobalt Gray equivalent (CGE), assuming a relative radiobiological effectiveness (RBE) of 1.1. The tumor was assumed to be free of infiltration of the optic disk or macula. The dose distribution was normalized so that ninety-five percent of the PTV received at least $100 \%$ of the prescribed dose; i.e., "D95" for the PTV was set to $60 \mathrm{CGE}$. Four fractions in one week were assumed to be used, which is the common practice in conventional proton therapy. The effects of the energy spread and beam diameter were investigated with 60 CGE kept for D95 in each calculation.

\section{Results}

In the first series of simulations, the effect of the energy spread of the beam on the dose distribution was investigated. Energy spread values of 5, 10, and 15 percent were simulated. The beam diameter was $2.5 \mathrm{~mm}$ in each case. Dose distributions are shown in Fig. 3. Dosevolume histograms (DVHs) are shown in Fig. 4, and simulation results are summarized in Table 1a. The table indicates that, at 5\% energy spread, the dose to the macula and optic disc was below the tolerance values for the case that we simulated. At $10 \%$ and $15 \%$, it is difficult to preserve critical structures located behind the distal edge of the target volume due to the elongated fall-off of the depth profile curve.

Target spot and beam characteristics are summarized in Table 1b. The table shows the effect of energy spread $(5 \%, 10 \%$, and $15 \%)$ on the depth spacing and the number of target spots with a constant beam diameter $(2.5 \mathrm{~mm})$. The depth spacing (the distance between target spots along a beam) is increased with increasing energy spread, whereas the number of target spots is decreased with increasing energy spread. 
In the second series, the effect of the beam diameter on the dose distribution was investigated. Beam diameter values of $1.25,2.5$, and $5 \mathrm{~mm}$ were simulated. The energyspread value for each case was 5\%. Dose distributions are shown in Fig. 5, and DVHs are shown in Fig. 6. Figure 5 illustrates that the volume of hot spots in the PTV increased when the beam diameter was increased from $1.25 \mathrm{~mm}$ to $2.5 \mathrm{~mm}$. The simulation results are summarized in Table 2a. The table indicates that a beam diameter of less than or equal to 2.5 $\mathrm{mm}$ would not exceed the tolerance doses for the case that we studied. At $5 \mathrm{~mm}$ beam diameter, the dose to the lens becomes significant.

Table $2 \mathrm{~b}$ indicates the effect of the beam diameter $(1.25 \mathrm{~mm}, 2.5 \mathrm{~mm}$, and $5 \mathrm{~mm})$ with a fixed energy spread of 5\%. The maximum and minimum values of the depth spacing were almost constant, as anticipated from the fixed energy spread. Also, the beam diameter affected the lateral spacing, as expected. The number of beams and target spots decreased significantly with an increase in the beam diameter. For a beam of $1.25 \mathrm{~mm}$, nearly a thousand target spots were generated by the planning software. Such a large number of target spots would likely require too much time to treat.

A histogram of particle energy for a typical treatment plan is displayed in Fig. 7. In general, more particles at the higher-energy end of the spectrum are necessary because more particles are targeted at deeper locations in forming the SOBP. The energy distribution is also affected by the target shape and the incident beam direction. The energy distribution is not smooth, partly due to the discrete proton energy values used for our calculations.

\section{Discussion}

Many parameters and parameter combinations (such as beam diameter, energy spread, lateral spacing, depth spacing, number of beams, and number of target spots) must be 
considered in assessing proton treatment of small superficial tumors. Realistically, some of the parameters may need to be predetermined in the clinical equipment because of mechanical or other limitations. In this study, we have simulated the effect of energy spread by using a fixed beam diameter $(2.5 \mathrm{~mm})$, and the effect of the beam diameter by using a fixed energy spread (5\%). These values were chosen because they seemed to be the most likely parameters delivered by an actual accelerator. A more thorough parameter survey is necessary for determination of the effects of every possible combination of beam parameters.

For reducing treatment times, it is desirable to reduce the number of target spots in a plan. However, there is a trade-off between the dose distribution and the number of target spots. Our results suggest that, if the beam energy and lateral spacing are predetermined, the energy spread and beam diameters must be chosen carefully with this in mind. The clinical significance of dose-volume statistics of the PTV and organs at risk must be determined for each patient.

The dose distributions shown here contain many hot spots (overdose areas). These are caused partially by the histogram normalization method, where $95 \%$ of the target volume is forced to receive at least $100 \%$ of the prescribed dose. Without normalization, cold spots were prevalent around the lateral and distal edges, especially when a large beam diameter or energy spread was used. The cold spots became prevalent when the distance from the tumor polygon edge and nearest target spot was relatively large. This resulted in DVH curves for the target volume are not as steep (selective) as they should have been. Several methods could be employed for improving the dose distributions, including increasing the number of Monte Carlo events, decreasing the space between target spots, and improving the optimization algorithm. Furthermore, we have used a fixed spacing factor of 0.5 in this study. Introduction of a variable spacing factor for each beam may further improve the homogeneity of dose 
distributions. The spacing factor should be smaller near the polygon edges to prevent cold spots while maintaining a reasonable total number of spots. These are topics for future study.

In this work, doses to the macula and to the optic disc were high and above the clinical limits in some cases. This is mainly because these structures are located directly behind the distal edge of the target volume. Proton spectra that are closer to being monoenergetic may improve the final clinical outcome. The effect of energy spread for deep-seated tumors such as prostate cancer may be different from that for shallow ocular diseases and is yet to be determined.

The required proton flux can be estimated as follows: Consider a shallow tumor volume of $1 \mathrm{cc}(1 \mathrm{gram})$. If the protons deposit an average energy of $50 \mathrm{MeV}$, then each proton delivers about $8 \times 10^{-12} \mathrm{~J}$ or $8 \times 10^{-9}$ Gy on average. Assuming an RBE of 1.1 , each proton delivers about $9 \times 10^{-9}$ CGE. A typical treatment course consists of 60 CGE delivered in four fractions on consecutive days. If the irradiation time is limited to 1 minute, then the accelerator must deliver 15 CGE per minute or .25 CGE per second. The accelerator must therefore produce about 30 million protons per second, or $4.8 \mathrm{pA}$. If such a delivery is carried out in $100 \mathrm{~Hz}$ repetitive pulsed laser shots, the required delivery is on the order of $3 \times 10^{5}$ protons per shot.

\section{Conclusion}

Our simulations show that a 2.5 -millimeter beam diameter and a 5\% percent energy spread can be considered as a starting point for ocular cases. The dose distributions suggest that there is merit to continuing such parameter studies and to considering further the potential for spot scanning proton sources.

\section{Acknowledgments}


This work was supported by the Core Research for Evolutional Science and Technology (CREST), the Japan Science and Technology Agency (JST), and the Special Coordination Fund (SCF) for Promoting Science and Technology commissioned by the Ministry of Education, Culture, Sports, Science and Technology (MEXT) of Japan. K. S. is a Takuma Scholar of PMRC.

\section{References}

1. Brenner DJ, Hall EJ. Secondary neutrons in clinical proton radiotherapy: a charged issue. Radiat. Oncol. 2008;86(2):165-70

2. Kanai T, Kawachi K, Kumamoto Y, Ogawa H, Yamada Y, Matsuzawa H. Spot scanning system for proton radiotherapy. Med. Phys. 1980;7 (4):365-9

3. Lomax AJ, Bohringer T, Bolsi A, Coray D, Emert F, Goitein G, et al. Treatment planning and verification of proton therapy using spot scanning: Initial experiences. Med. Phys. 2004;31 (11):3150-7

4. Lomax AJ, Boehringer T, Coray A, Egger E, Goitein G, Grossmann M, et al. Intensity modulated proton therapy: A clinical example. Med. Phys. 2000;28 (3):317-24

5. Tajima T. Prospect for compact medical laser accelerators. J. Jpn. Soc. Ther. Radiol. Oncol. 1997; 9 (Suppl 2), 83

6. Malka V, Sven F, Lefebvre E, d'Humieres E, Ferrand R. Practicability of protontherapy using compact laser systems. Med Phys. 2004;Volume 31 Issue 6:1587-92

7. Ma CM, Maughan RL. Point/Counterpoint: Within the next decade conventional cyclotrons for proton radiotherapy will become obsolete and replaced by far less expensive machines using compact laser systems for the acceleration of the protons. Med Phys. 2006; 33 (3):571-3. 
8. Bulanov SV, Esirkepov T, Khoroshkov VS, Kuznetsov AV, Pegoraro F. Oncological hadrontherapy with laser ion accelerators. Phys. Lett. A. 2002; 299:240-7

9. Agostinelli S, Allisonas J, Amakoe K, Apostolakisa J, Araujo H, et al. Geant4 - a simulation toolkit. Nuclear Instruments and Methods in Physics Research A. 2003;506:250-303

10. Carrier J, Archambault L, Beaulieu L, Roy R. Validation of Geant4, an object-oriented Monte Carlo toolkit, for simulations in medical physics. Med. Phys. 2004;31 (3):484-92

11. Jiang H, Paganetti H. Adaptation of Geant4 to Monte Carlo dose calculations based on CT data. Med. Phys. 2004;31(10):2811-18

12. Sutherland K, Miyajima S, Date H. A simple parallelization of Geant4 on a PC cluster with static scheduling for dose calculations. First European Workshop on Monte Carlo Treatment Planning, Journal of Physics: Conference Series. 2007; 74012020

13. Schneider W, Bortfeld T, Schlegel W. Correlation between CT numbers and tissue parameters needed for Monte Carlo simulations of clinical dose distributions. Phys. Med. Biol. 2000; 45:45978

14. Iwase H, Niita K, Nakamura T. Development of a general-purpose particle and heavy ion transport Monte Carlo code. Journal of Nuclear Science and Technology. 2002;Vol. 39, No. 11:1142-51

15. Niita K, Sato T, Iwase H, Nose H, Nakashima H and Sihver L. PHITS-a particle and heavy ion transport code system. Radiation Measurements. 2006; 41 1080-1090

16. Dendale R, et al. Proton beam radiotherapy for uveal melanoma: results of curie institute-orsay proton therapy center (ICPO). Int. J. Radiation Oncology Biol. Phys. 2006; Vol. 65, No. 3:780-87

\section{Figure captions}




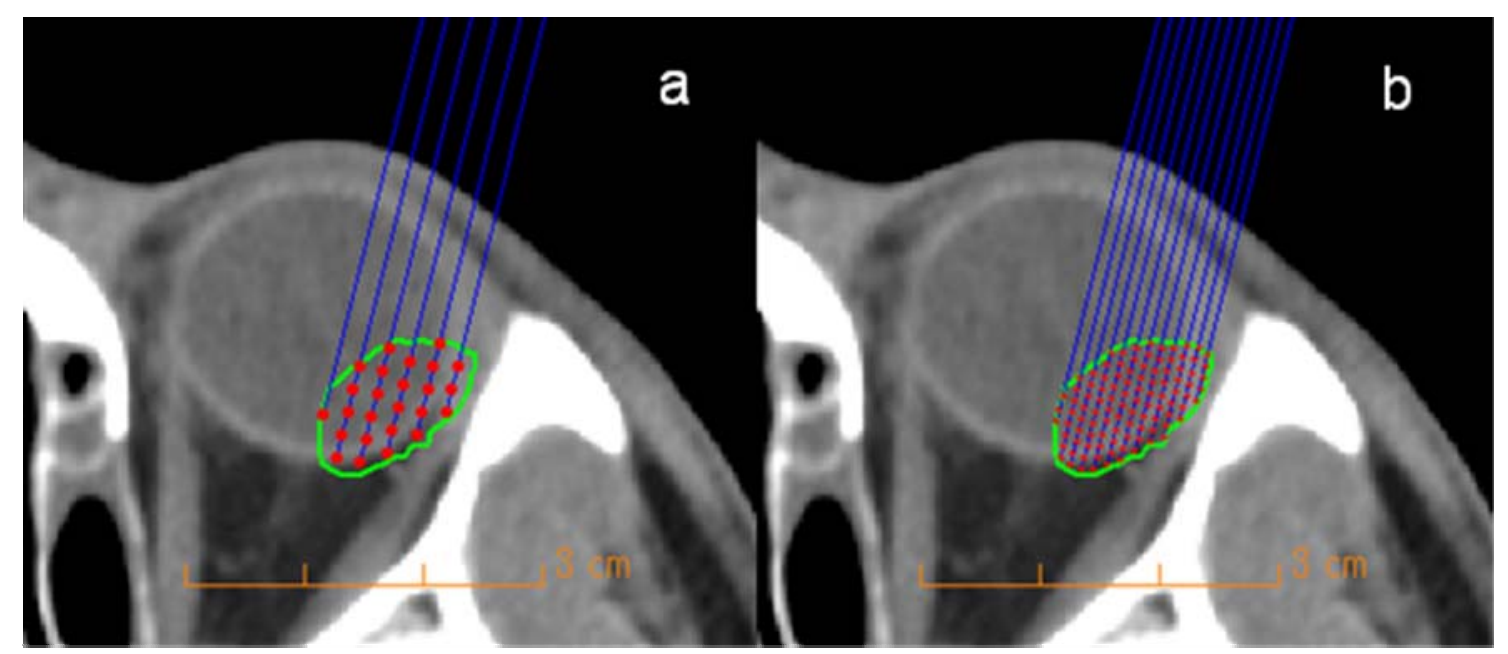

Figure 1. Effect of beam parameters on target spot placement and beam spacing. Blue lines are beams. The green polygon is the planned target volume (PTV). Red dots are target spots. (a) $5 \mathrm{~mm}$ beam diameter and $10 \%$ energy spread. (b) $1.25 \mathrm{~mm}$ beam diameter and $0 \%$ energy spread, for which more target spots are generated.

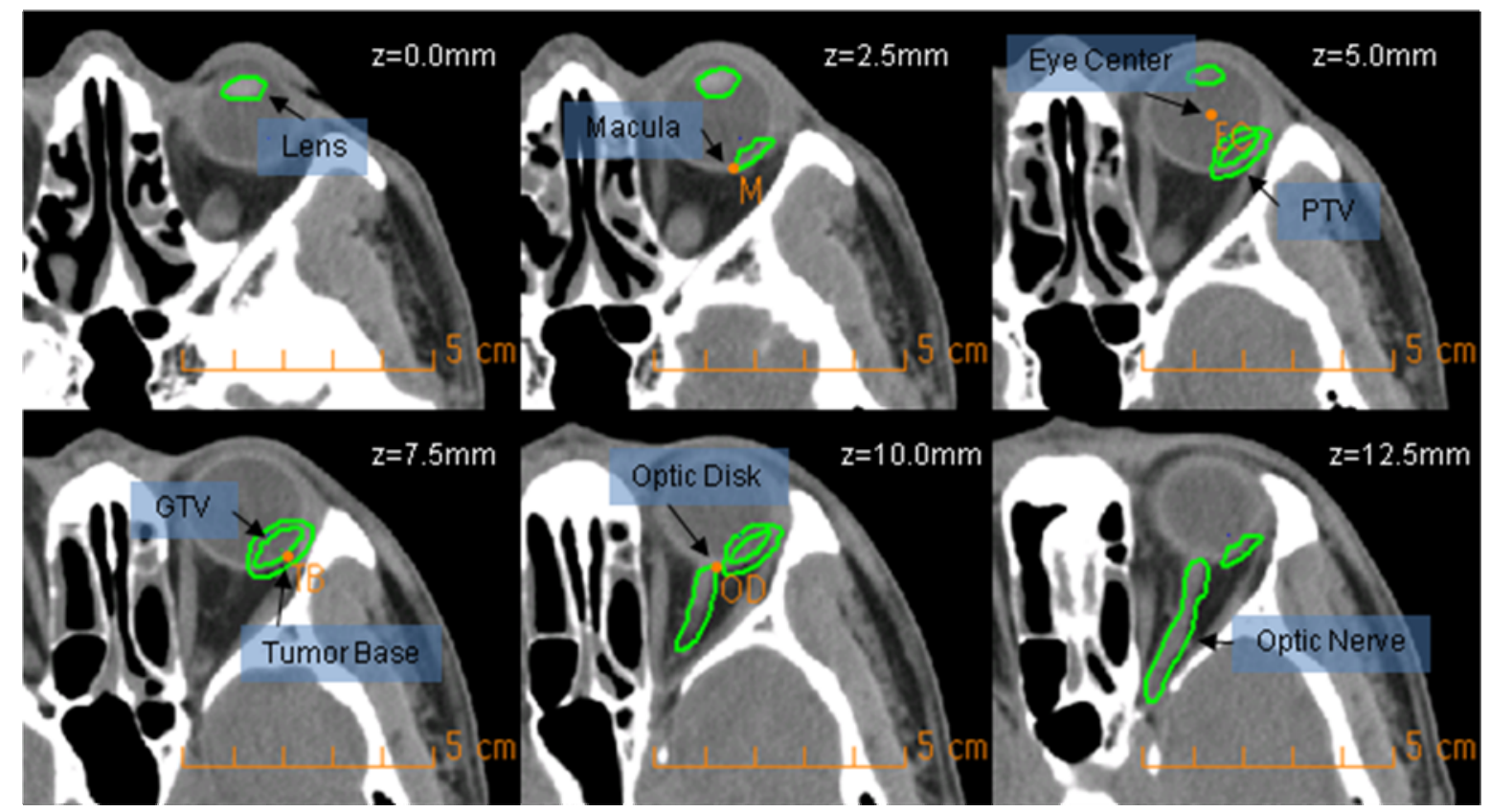

Figure 2. Target polygons and organs at risk (OARs) used for this experiment. The inner target polygon is the GTV. The GTV is generated automatically by specifying the location of the tumor base and eye center. The size of the GTV is determined by specifying the basal 
diameter and tumor height. The outer target polygon is the PTV (GTV plus $2 \mathrm{~mm}$ margin). OARs include the lens, optic nerve macula, and optic disk.

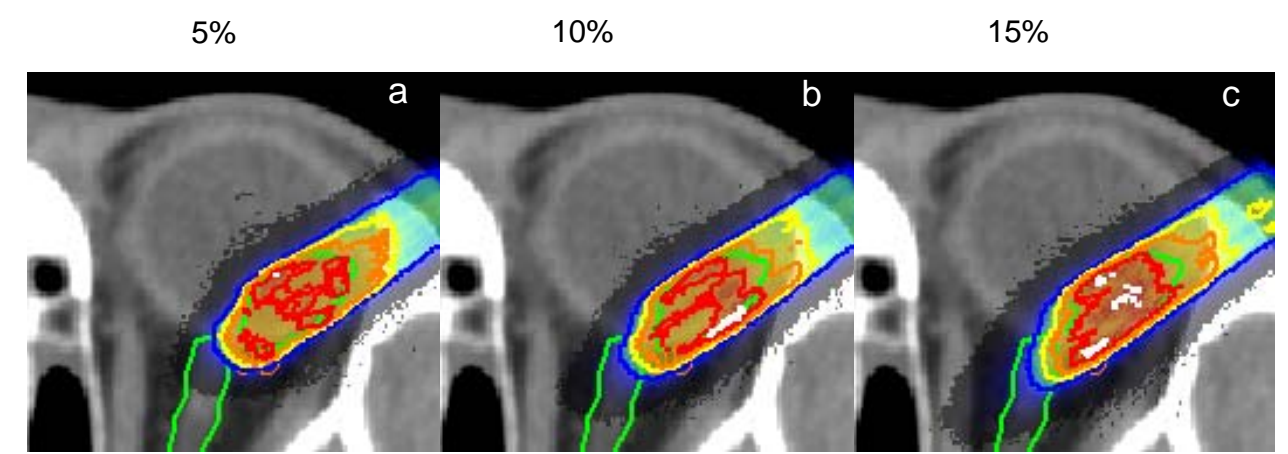

Figure 3. Dose distributions for various values of energy spread. $5 \%$ (a), $10 \%(\mathrm{~b})$, and $15 \%$ (c). Isodose lines are $125 \%$ of prescribed dose (75 CGE) white, 110\% (66 CGE) red, 90\% (54 CGE) orange, 75\% (45 CGE) yellow, and 50\% (30 CGE) blue. In all cases a $2.5 \mathrm{~mm}$ beam width was used. 


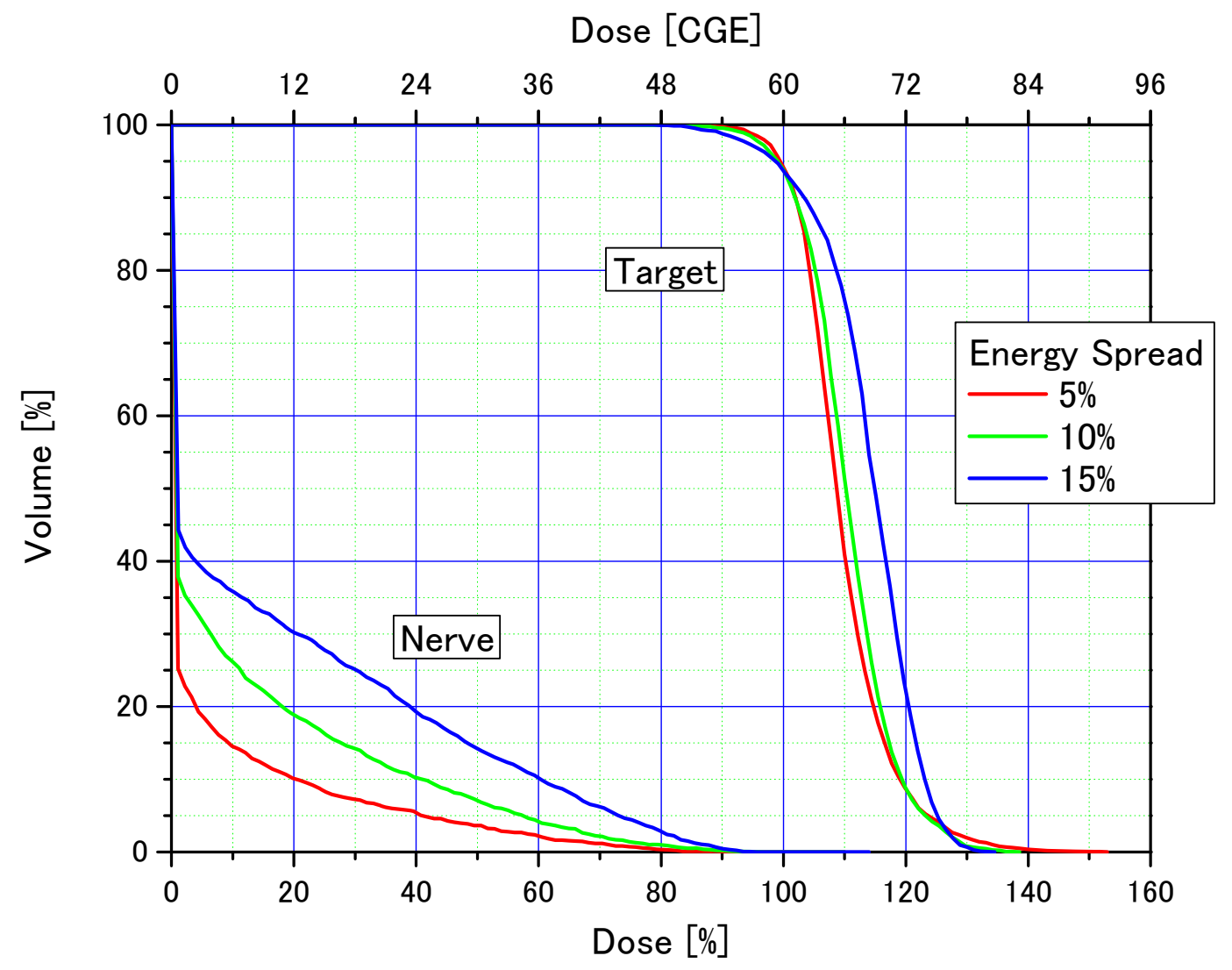

Figure 4. DVH of PTV and optic nerve for various energy spread values. In all cases a 2.5 mm beam diameter was used.

$1.25 \mathrm{~mm}$

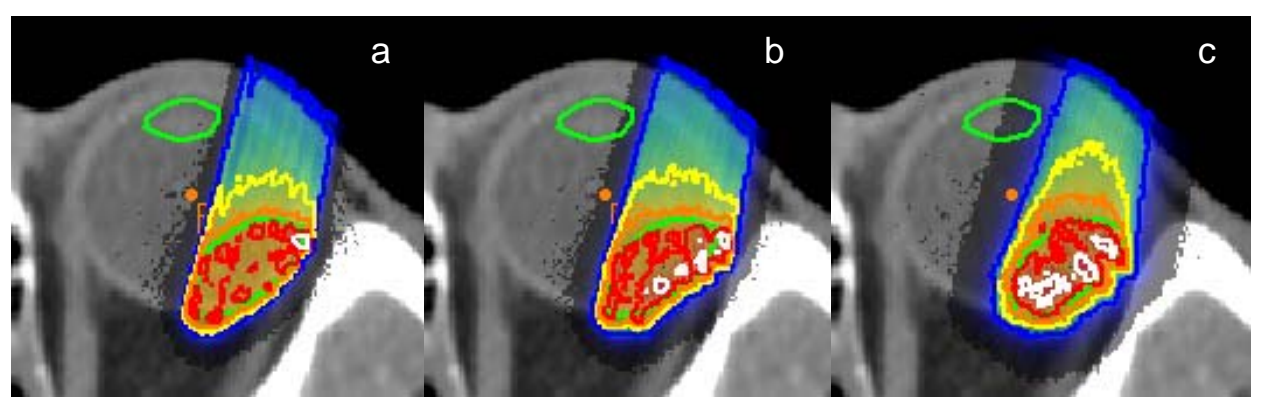

Figure 5. Dose distribution for various values for beam diameter, $1.25 \mathrm{~mm}$ (a) $2.5 \mathrm{~mm}$ (b), and $5 \mathrm{~mm}(\mathrm{c})$. Isodose lines are $125 \%$ of prescribed dose (75 CGE) white, $110 \%$ (66 CGE) 
red, 90\% (54 CGE) orange, 75\% (45 CGE) yellow, and 50\% (30 CGE) blue. In all cases we used 5\% energy spread.

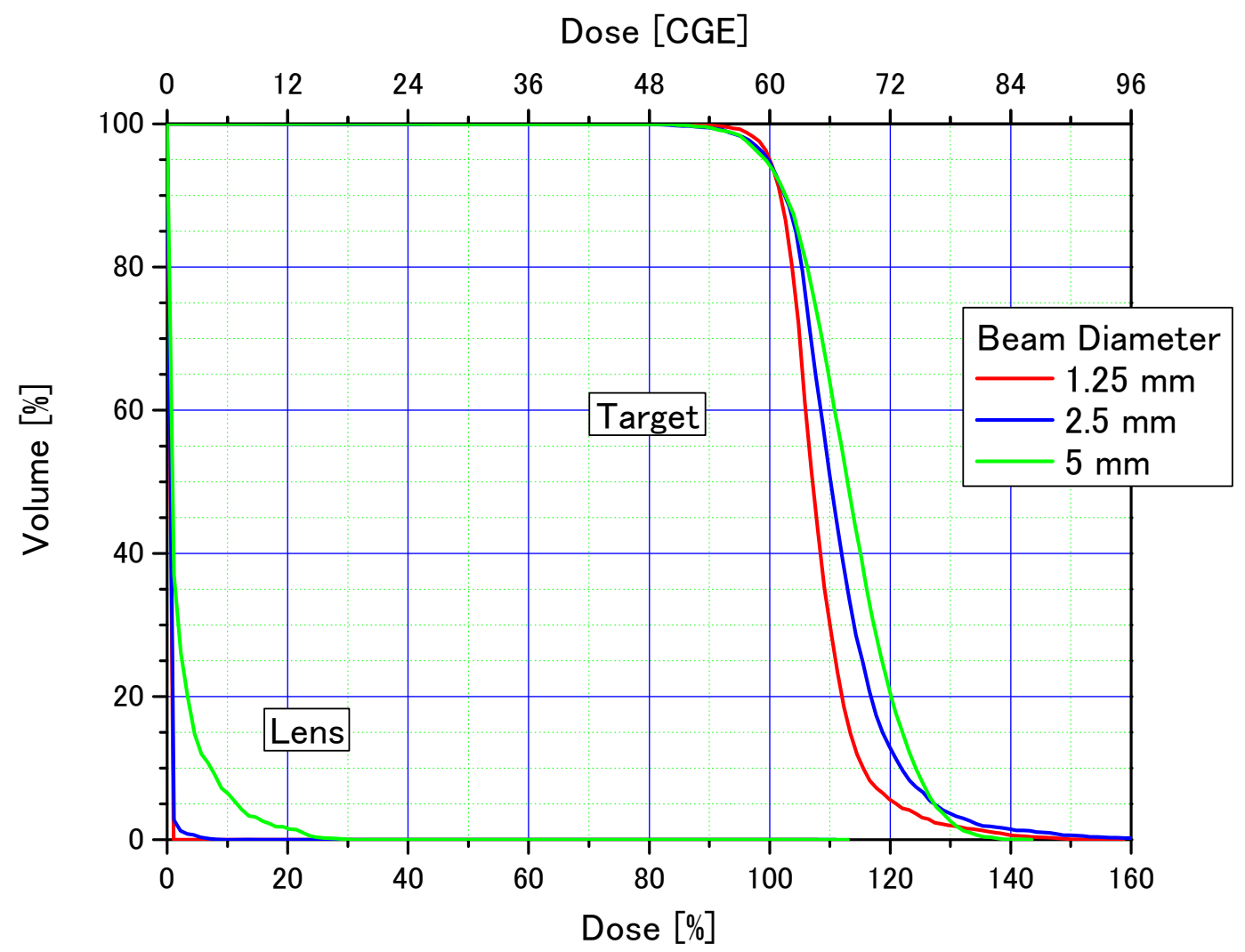

Figure 6. DVH for PTV and optic nerve computed with various values for beam diameter. In all cases we used 5\% energy spread. 


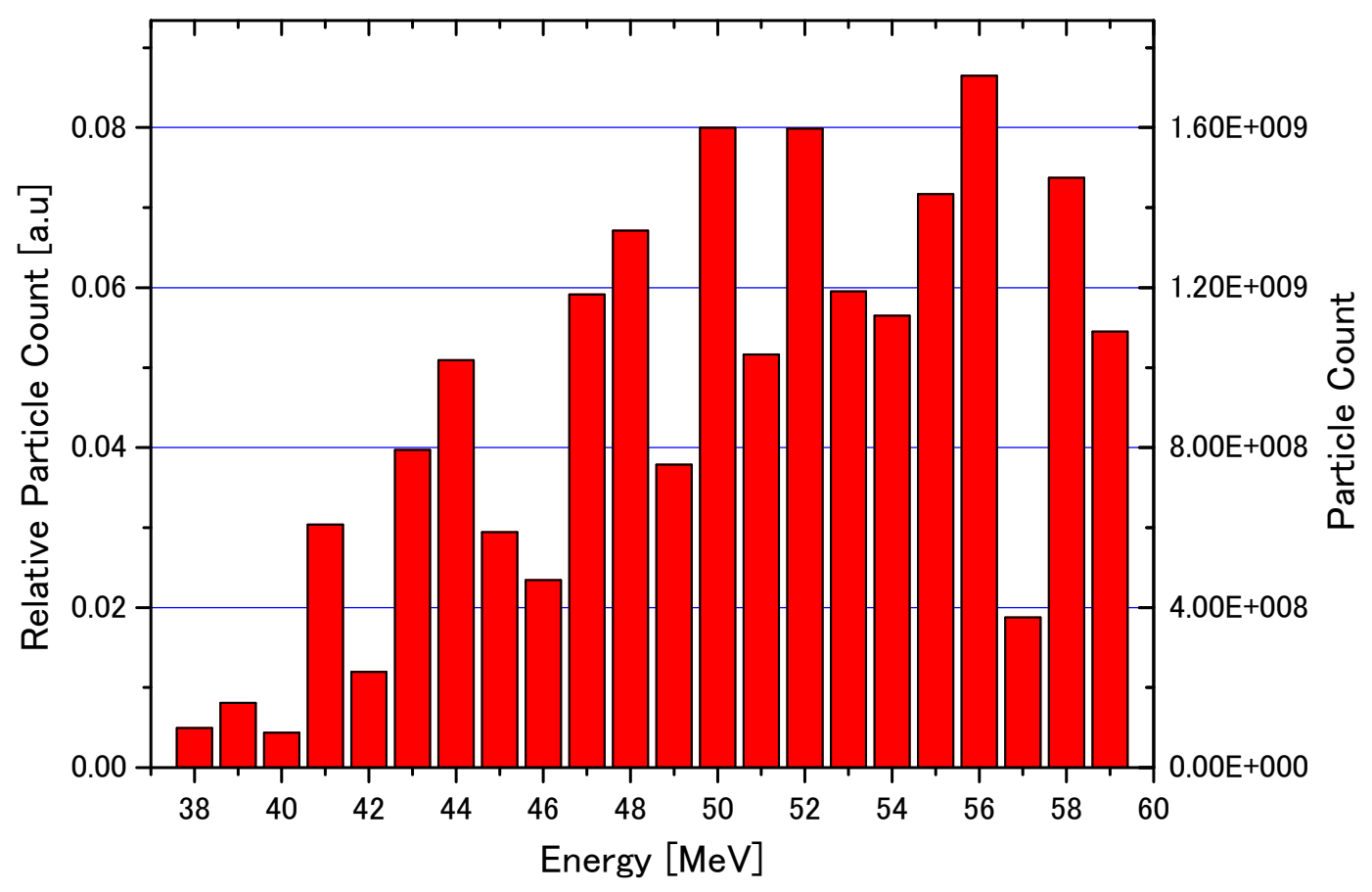

Figure 7. A typical histogram of particle energy levels.

Table 1a. Dosimetric Characteristics - Energy Spread.

\begin{tabular}{lrrr}
\hline Energy Spread & $5 \%$ & $10 \%$ & $15 \%$ \\
\hline Retina $\geq 45$ CGE & $1 \%$ & $2 \%$ & $5 \%$ \\
Lens $\geq 10$ CGE & $0 \%$ & $0 \%$ & $0 \%$ \\
Optic Nerve $\geq 12$ CGE & $10 \%$ & $20 \%$ & $31 \%$ \\
Dose at Macula ( $\geq 30$ CGE) & $27(\mathrm{OK})$ & $36(\mathrm{NG})$ & $42(\mathrm{NG})$ \\
Dose at Optic Disc $(\geq 12$ CGE) & $11(\mathrm{OK})$ & $1.8(\mathrm{OK})$ & $3.3(\mathrm{OK})$ \\
V95 $^{\text {a }}$ & $98 \%$ & $98 \%$ & $97 \%$ \\
\hline
\end{tabular}

${ }^{a}$ Percent volume of PTV which received $95 \%$ of the prescribed dose (57 CGE)

Table 1b. Target Spot and Beam Characteristics - Energy Spread ${ }^{\mathrm{a}}$.

\begin{tabular}{lrrr}
\hline Energy Spread & $5 \%$ & $10 \%$ & $15 \%$ \\
\hline Lateral Spacing [mm] & 1.3 & 1.3 & 1.3 \\
Depth Spacing Min [mm] & .6 & 1.7 & 3.1 \\
Depth Spacing Max [mm] & 2.8 & 5.8 & 9.1 \\
Number of Beams & 38 & 38 & 38 \\
Number of Target Spots & 239 & 102 & 65 \\
\hline
\end{tabular}

${ }^{a}$ Beamlet diameter $2.5 \mathrm{~mm}$ for each case 
Table 2a. Dosimetric Characteristics - Beam Diameter.

\begin{tabular}{lrrr}
\hline Beam Diameter & $1.25 \mathrm{~mm}$ & $2.5 \mathrm{~mm}$ & $5 \mathrm{~mm}$ \\
\hline Retina $\geq 45 \mathrm{CGE}$ & $0 \%$ & $0 \%$ & $1 \%$ \\
Lens $\geq 10 \mathrm{CGE}$ & $0 \%$ & $0 \%$ & $6 \%$ \\
Optic Nerve $\geq 12 \mathrm{CGE}$ & $0 \%$ & $1 \%$ & $4 \%$ \\
Dose at Macula ( $\geq 30 \mathrm{CGE})$ & $13(\mathrm{OK})$ & $13(\mathrm{OK})$ & $36(\mathrm{NG})$ \\
Dose at Optic Disc ( $\geq 12 \mathrm{CGE})$ & $0.8(\mathrm{OK})$ & $3.0(\mathrm{OK})$ & $18(\mathrm{NG})$ \\
V95 $^{\mathrm{a}}$ & $99 \%$ & $98 \%$ & $98 \%$ \\
\hline
\end{tabular}

${ }^{\mathrm{a}}$ Percent volume of CTV which received $95 \%$ of the prescribed dose (57 CGE)

Table 2b. Spot and Beam Characteristics - Beam Diameter ${ }^{\mathrm{a}}$.

\begin{tabular}{lrrr}
\hline Beam Diameter & $1.25 \mathrm{~mm}$ & $2.5 \mathrm{~mm}$ & $5 \mathrm{~mm}$ \\
\hline Lateral Spacing [mm] & .7 & 1.4 & 2.6 \\
Depth Spacing Min [mm] & .7 & .7 & .6 \\
Depth Spacing Max [mm] & 2.4 & 2.4 & 2.3 \\
Number of Beams & 206 & 57 & 21 \\
Number of Target Spots & 918 & 233 & 87 \\
\hline
\end{tabular}

${ }^{a}$ Energy spread 5\% for each case 